\title{
Dynamic Resistance Measurement of a Four-tape YBCO Stack in a Perpendicular Magnetic Field
}

\author{
Z. Jiang, W. Zhou, C. W. Bumby, M. Staines, Q. Li, R. A. Badcock, N. J. Long, and, J. Fang
}

\begin{abstract}
Dynamic resistance occurs when HTS (high temperature superconductor) coated conductors carry dc current under ac magnetic field. This dissipative effect can play critical role in many HTS applications. Here, we report on dynamic resistance measurements of a four-tape YBCO stack comprising $4 \mathrm{~mm}$-wide coated conductors which experience an applied ac perpendicular magnetic field with an amplitude of up to $100 \mathrm{mT}$. Each tape within the stack carries the same dc current. The magnetic field amplitude, the frequency of the magnetic field, and the dc current magnitude are varied to investigate the influence of these parameters on the dynamic resistance. We find that the threshold field of the stack is significantly larger than that of a single tape when dc current is small, which we attribute to coherent shielding effects from circulating currents present in each wire in the stack.
\end{abstract}

Index Terms - Coated conductor, dynamic resistance, stack

\section{INTRODUCTION}

I $\mathrm{N}$ many HTS (high- $T_{\mathrm{c}}$ superconductor) applications, such as rotating machines, magnets, flux pumps, and Superconducting Energy Storage Systems (SMES), HTS wires carry dc transport current under external ac magnetic fields [1]-[7]. For the small amplitude of the external magnetic field, magnetic field penetration occurs only at both sides of a superconductor, and dc current can flow in the central region of the conductor without associating any energy dissipation. However, when the amplitude of the external magnetic field is larger than a threshold amplitude, magnetic flux enters the conductor from one side, traverses across the central region of the conductor where dc current flows, leaves the conductor from the other side of the conductor, and dynamic resistance occurs during this process [8]-[12]. Dynamic resistance is a form of hysteretic ac loss, and results in a parasitic heat load on the cryogenic system. For some high current applications, it is essential to assemble multiple HTS wires into stacks $[13,14]$. Therefore, it is important to understand the dynamic loss characteristics of HTS stacks carrying dc current when exposed to an external ac magnetic field.

In previous works, we have measured dynamic resistance in single coated conductor wires exposed to perpendicular magnetic fields. We have developed a simple analytical approach

Manuscript received September 19, 2017. This work was supported by New Zealand MBIE Contract No. RTVU1304. (Corresponding author: Zhenan Jiang)

Z. Jiang, C. W. Bumby, M. Staines, R. A. Badcock, and N. J. Long are with the Robinson Research Institute, Victoria University of Wellington, PO Box 33436, Lower Hutt 5046, New Zealand. (e-mail: zhenan.jiang@vuw.ac.nz).

Q. Li is with School of Engineering, University of Edinburgh, Faraday Building, The King’s Buildings, Edinburgh EH9 3JL, UK (e-mail:quan.li@ed.ac.uk ). which predicts the dynamic resistance which occurs in this case [15]-[17]. However, there have been no reports of dynamic resistance measurements in HTS stacks. In this work, we report on dynamic resistance measurements of a four-tape YBCO stack comprising $4 \mathrm{~mm}$-wide coated conductors when exposed to ac perpendicular magnetic field. Each tape within the stack carries the same dc current, achieved by serial connection of the tapes. The magnetic field amplitude, the frequency of the magnetic field, and the dc current magnitude have been varied in order to investigate the influence of these parameters on the dynamic resistance. From our experimental results, we have extracted values for the threshold field and field-dependence of the measured dynamic resistance, for each experimental condition considered. We then compare these values with analytical expressions which describe the expected behavior of a single coated conductor wire.

\section{EXPERIMENTAL METHOD}

Fig. 1 shows a schematic of the experimental arrangement used in this work to measure dynamic resistance of a four-tape YBCO stack at $77 \mathrm{~K}$. An ac magnet comprising two vertically stacked sets of racetrack coils was used to produce ac magnetic field amplitudes at the sample of up to $100 \mathrm{mT}$. Two different ac frequencies were employed [18]. The HTS stack was mounted on the flat machined surface of a G-10 sample holder and placed in the center of the magnet. The direction of the magnetic field was adjusted in order to ensure it was perpendicular to the wide-face of the stack. This was achieved through adjusting sample alignment to maximize the voltage signal in a single-turn pick-up coil on the surface of the sample holder. The coated conductor wires employed in this work were SuperPower Inc. copper stabilized SCS4050 wires [19]. Each tape was insulated from the other tapes in the stack using Kapton tapes and sheets, such that the distance between the neighboring superconductor layers was $\sim 300 \mu \mathrm{m}$. Two sets of voltage taps were used as shown in Fig. 1. In the first set, a spiral loop was attached around each conductor. In the second set, two voltage taps were mounted on the center of each conductor and the four-

W. Zhou and J. Fang are with School of Electrical Engineering, Beijing Jiaotong University, No. 3 Shangyuncun, Haidian District, Beijing 100044, the People's Republic of China. (email: jfang@bjtu.edu.com) 
pairs of voltage leads were extended to the outside after meeting at the center point of each conductor [20]. The distance between the voltage taps was $5 \mathrm{~cm}$. Subsequent analysis of the experimental data showed that the voltage signals from the two sets of voltage taps were identical. The voltage signals from the spiral loops are shown throughout this work. The conductors in the stack were connected in series to ensure that the same transport current was flowing in each wire. The current leads between each wire were arranged so as to avoid magnetic coupling between the magnet and dc current circuits (as shown in the figure). The dc voltage output from the voltage taps was measured using a dc Hewlett Packard 34420A nanovolt-meter. The sample integration time (NPLC) of the nanovolt-meter was set at 10 power line cycles.

The $I_{\mathrm{c}}$ values of the four conductors before and after assembling the stack were measured, and are listed in Table I. The measured $I_{\mathrm{c}}$ values were reduced in the stack environment, due to the superposition of magnet fields generated by the other conductors in the stack [21].

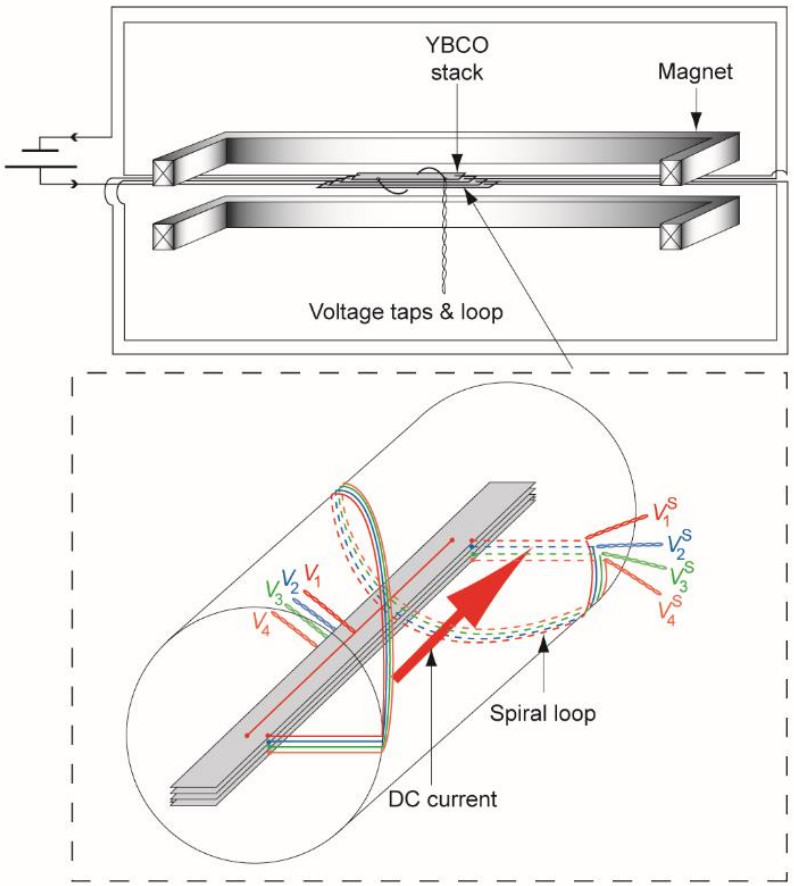

Fig. 1. Experimental set-up for dynamic resistance measurement and voltage tap arrangement.

TABLE I

$I_{C}$ VALUES OF THE FOUR CONDUCTORS COMPRISING THE STACK

\begin{tabular}{lccccl}
\hline \hline & T-1 & T-2 & T-3 & T-4 & Average \\
\hline \hline Self-field $I_{\mathrm{c}}(\mathrm{A})$ & 96.6 & 96.6 & 96.0 & 96.5 & 96.4 \\
$I_{\mathrm{c}}$ after assembling (A) & 82.3 & 83.8 & 83.0 & 85.1 & 83.6 \\
\hline \hline
\end{tabular}

\section{EXPERIMENTAL RESULTS}

Figs 2 and 3 show the dynamic resistance, $R_{\text {dyn, values for }}$ each tape within the stack measured at two different frequency and current values. In these figures $R_{\text {dyn }}$ is normalized by frequency and plotted as a function of the magnetic field amplitude. It can be seen that in each case, dynamic resistance is negligible until the threshold magnetic field amplitude is exceeded. This is similar to the behavior observed in single coated conductors [15]. There is no obvious difference between the results at $67.89 \mathrm{~Hz}$ and $87.65 \mathrm{~Hz}$ for dc current values of $8.3 \mathrm{~A}$ and $24.8 \mathrm{~A}$. This reflects the hysteretic nature of dynamic resistance in the stack. Similar results were also obtained at additional dc current values which are not shown in this paper.
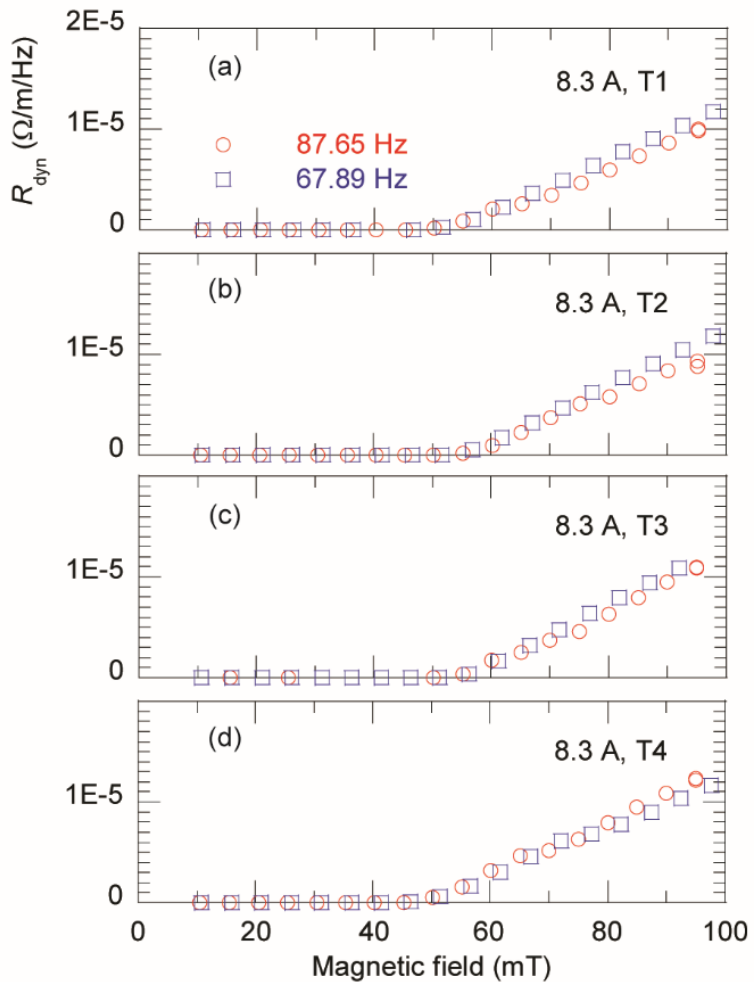

Fig. 2. Measured dynamic resistance in each conductor of the YBCO stack at 8.3 A for $67.89 \mathrm{~Hz}$ and $87.65 \mathrm{~Hz}$, (a) T-1, (b) T-2, (c) T-3, and (d) T-4.
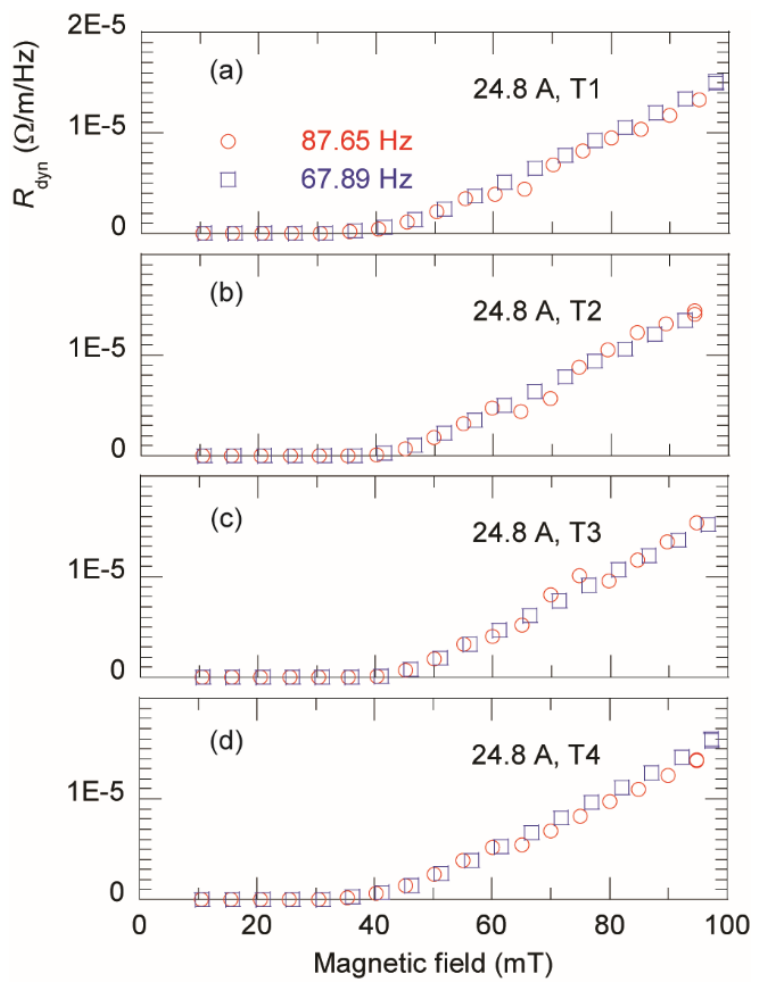

Fig. 3. Measured dynamic resistance in each conductor of the YBCO stack at 24.8 A for $67.89 \mathrm{~Hz}$ and $87.65 \mathrm{~Hz}$, (a) T-1, (b) T-2, (c) T-3, and (d) T-4. 
Figure 4 shows the measured dynamic resistance data obtained from each tape at $67.89 \mathrm{~Hz}$, and using five different current values. The dynamic resistance values measured at each position within the stack are observed to very similar in all cases. This enables a single threshold field to be identified for the entire stack at each dc current value. These values were extracted from the $x$-axis intercept of linear fits of the measured data. The threshold values for the stack decrease with increasing dc current values. This trend is similar to that observed in dynamic resistance measurements of single coated conductor tapes $[15$, $16]$.
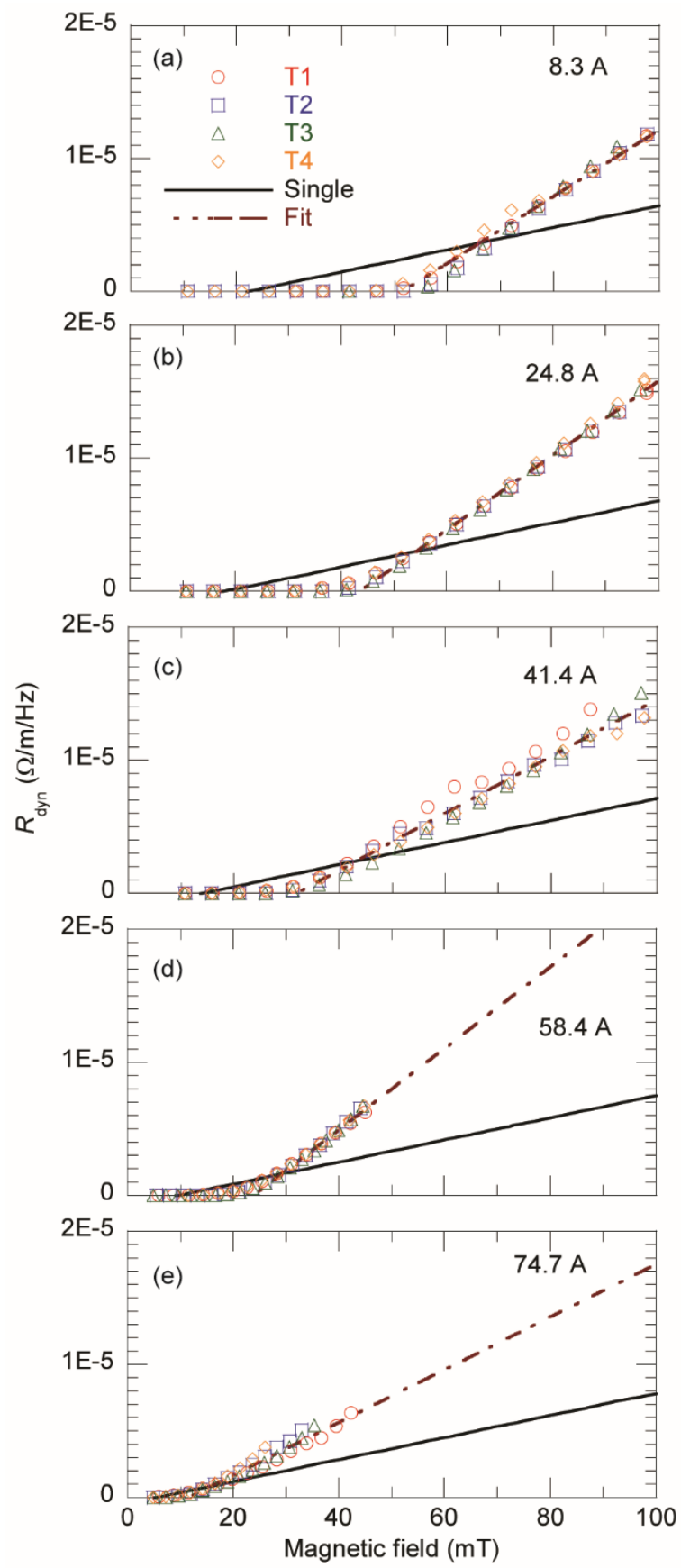

Fig. 4. Dynamic resistance data in each tape for five different current values at $67.89 \mathrm{~Hz}$, (a) $8.3 \mathrm{~A}$, (b) $24.8 \mathrm{~A}$, (c) $41.4 \mathrm{~A}$, (d) $58.4 \mathrm{~A}$, and (e) $74.7 \mathrm{~A}$.

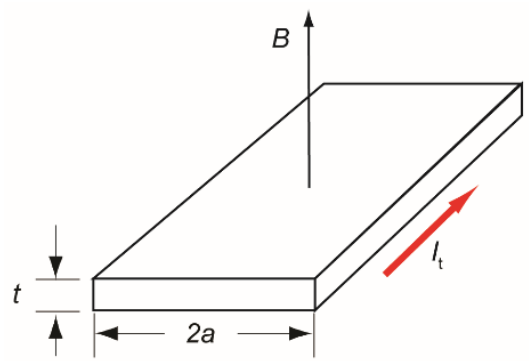

Fig. 5. Schematic of a superconducting strip carrying DC current under an ac perpendicular magnetic field.

In our previous work, we have shown that dynamic resistance in coated conductors exposed to perpendicular magnetic fields, can be well described by an analytical equation [15].

We consider a superconducting strip with a thickness, $t$, and a width, $2 a$, as shown in in Figure 5. This strip carries a dc current, $I_{\mathrm{t}}$, and experiences an applied ac perpendicular magnetic field, of amplitude, $B_{\mathrm{a}}$. The dynamic resistance per unit length per cycle $R_{\mathrm{dyn}}$, is then given by [15],

$$
\frac{R_{\mathrm{dyn}}}{f l}=\frac{4 a}{I_{\mathrm{c} 0}}\left(B_{\mathrm{a}}-B_{\mathrm{th}}\right)
$$

where, $I_{\mathrm{c} 0}$ is the self-field critical current of the conductor, $l$ is the distance between the two voltage taps, and $f$ is the frequency of the applied magnetic field. The threshold magnetic field, $B_{\text {th }}$ is given by,

$$
B_{\mathrm{th}}=B_{\mathrm{ep}}\left(1-\frac{I_{\mathrm{t}}}{I_{\mathrm{c} 0}}\right)
$$

where $\left(1-I_{\mathrm{t}} / I_{\mathrm{c} 0}\right)$ is the filling portion of dc current and $B_{\mathrm{ep}}$ is effective penetration field $[13,15]$, which is equivalent to the field at the maxima of the Brandt and Indenbom equation for ac magnetization loss in a strip under perpendicular magnetic field [22]. This maxima field is obtained by analytical methods as, $B_{\mathrm{ep}}=2.4642 \mu_{0} t J_{\mathrm{c} 0} / \pi[15,16]$, where $J_{\mathrm{c} 0}$ is defined as $I_{\mathrm{c} 0} /(t \times 2 a)$.

Figure 4 shows the calculated dynamic resistance value obtained from equations (1) and (2), for a single coated conductor which has a self-field $I_{\mathrm{c}}$ value of $96.4 \mathrm{~A}$ (the averaged self-field $I_{\mathrm{c}}$ of the conductors in Table I). We denote this calculated value as 'Single' in the figure. Interestingly, we see that the measured $B_{\text {th }}$ values for the stack are significantly larger than the single conductor. We attribute this to a shielding effect from circulating current present in each wire in the stack [13, 14]. This effect is more significant when the dc current value is small, and becomes weaker with increasing dc current. This is to be expected, as larger transport currents in each tape leave occupy a greater fraction of the wire's total current-carrying capacity, thus reducing the total shielding currents flowing in each tape. Another notable observation is that the gradient values of the linear fits $\left(\mathrm{d} R_{\mathrm{dyn}} / \mathrm{d} B_{\mathrm{a}}\right)$ for each tape of the stack are much bigger than expected for a single conductor. This might be due to $I_{\mathrm{c}}(B)$ dependence of the wires in the stack. Such effects have previously 
been observed in the dynamic resistance of single BSCCO wires under parallel magnetic fields [9].

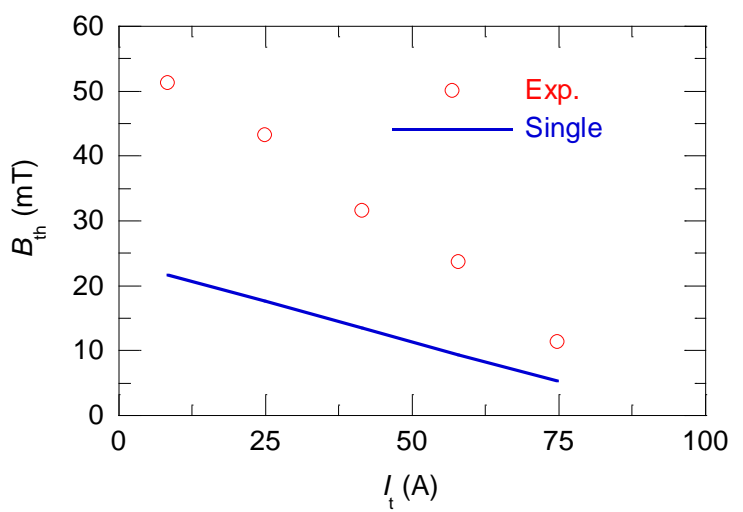

Fig. 6. Comparison of the experimentally obtained threshold field values, $B_{\text {th }}$ values for the stack and the analytically obtained $B_{\text {th }}$ values for the single conductor from Eq. (2).

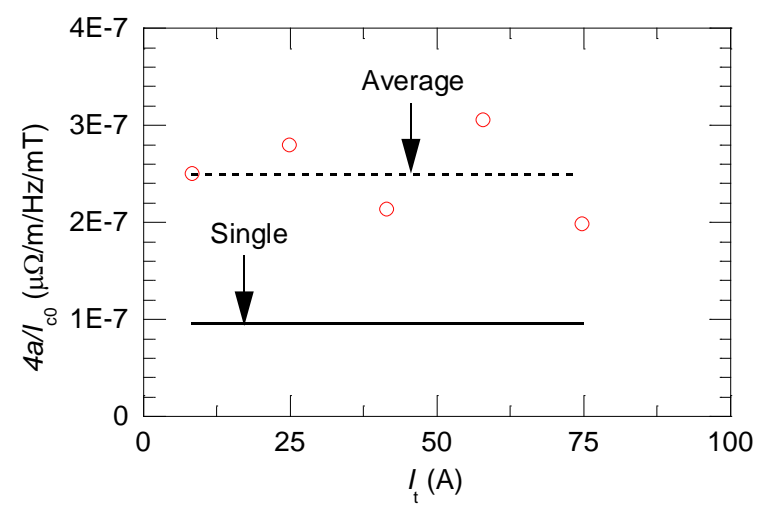

Fig. 7. Comparison of the experimentally obtained gradient of linear fits of the stack $\left(\mathrm{d} R_{\mathrm{dyn}} / \mathrm{d} B_{\mathrm{a}}\right)$ and the theoretical gradient value of $4 a / I \mathrm{c} 0$ for the single conductor from Eq. (1).

Figure 6 compares the experimentally obtained $B_{\text {th }}$ values for the stack, and calculated $B_{\text {th }}$ values for the single conductor (eq. (2), at various different dc current values. once again this clearly illustrates that the, $B_{\mathrm{th}}$ values of the stack are bigger than those for a single conductor throughout the range of current values investigated, due to the shielding effect. At $I_{\mathrm{t}}=8.4 \mathrm{~A}$, the $B_{\mathrm{th}}$ value for the stack is approximately 2.5 times that of a single conductor. However the $B_{\text {th }}$ values in the stack decrease at a slightly faster rate than those for the single conductor, such that at $I_{\mathrm{t}}=71.4 \mathrm{~A}$, the $B_{\mathrm{th}}$ value of the stack is approximately 2 times that of the single conductor.

In Fig. 7, the experimentally obtained values for $\mathrm{d} R_{\mathrm{dyn}} / \mathrm{d} B_{\mathrm{a}}$ of the stack are plotted, together with the theoretical gradient value of $4 a / I c 0$ for the single conductor from Eq. (1). Values are plotted versus the dc current value, and there is some scattering about the average gradient value. We attribute this to imperfect fitting of the composite data shown in Fig. 4. The averaged gradient value in the figure is more than 2.5 times the theoretical value for the single conductor. The origin of this increased gradient is not entirely clear at this time, and detailed electromagnetic field analysis will be required in order to probe this further.
In previous works [23, 24], Lahtinen et al carried out FEM AC loss simulations using CSM (critical state model) and ECM (eddy current model), when HTS conductors carry dc current under alternating external magnetic field. CSM assumes infinite $n$ value while ECM utilizes $E$ - $J$ power-law relationship. They pointed out that CSM exhibits better accuracy than ECM for dynamic resistance simulation, while ECM shows better agreement with experiment than CSM for magnetization loss in a conductor with dc current. Therefore, limitations of both models need to be considered when implementing numerical simulations for these conditions.

Dynamic resistance measurement in stacks carrying dc current super-imposed with ac current will be our next target [3].

\section{CONCLUSION}

We have measured the dynamic resistance under an ac perpendicular magnetic field, of a four-tape YBCO stack comprising $4 \mathrm{~mm}$-wide YBCO coated conductors each carrying same dc transport current.

Frequency-normalized values of the dynamic resistance were obtained at two different frequencies and observed to be identical at all currents and fields studied. This confirms the hysteretic nature of the dynamic resistance measured in the stack.

Threshold field values for the stack were found to be much larger than those expected for a single coated conductor. We attribute this to a superimposed shielding field arising from the magnetization currents present in each wire in the stack. This shielding effect becomes weaker with increasing dc transport current, as the circulating currents supported within each conductor are reduced.

The gradient, $\mathrm{d} R_{\mathrm{dyn}} / \mathrm{d} B_{\mathrm{a}}$, of the linear fits to the composite data for the stack is found to be more than twice that expected for a single coated conductor wire. The reason for this is unclear at present. One possibility is that it may be due to the $I_{\mathrm{c}}(B)$ dependence of the conductors in the stack. A numerical electromagnetic analysis of flux motion within the stack is now required, to provide detailed understanding of these experimental results.

\section{ACKNOWLEDGMENT}

The authors thank Gennady Sidorov for preparing some experimental components.

\section{REFERENCES}

[1] S. S. Kalsi, Applications of high temperature superconductors to electric power equipment (IEEE Press, New Jersey, 2011) pp.96.

[2] P. J. Masson, D. S. Soban, E. Upton, J. E. Pienkos, and C. A. Luongo, "HTS motors in aircraft propulsion: design considerations," IEEE Trans. Appl. Supercond. vol. 15, pp. 2218 - 2221, 2005.

[3] E. Pardo, "Modeling of AC loss in coils made of thin tapes under DC bias current," IEEE Trans. Appl. Supercond. vol. 24, 2013, Art. no. 4700105.

[4] Z. Jiang, K. Hamilton, N. Amemiya, R. A. Badcock, and C. W. Bumby, "Dynamic resistance of a high- $T_{\mathrm{c}}$ superconducting flux pump," Appl. Phys. Lett, vol. 105, Sep. 2014, Art. no. 112601. 
[5] J. Geng and T. A. Coombs, "Mechanism of a high-Tc superconducting flux pump: Using alternating magnetic field to trigger flux flow," Appl. Phys. Lett. vol. 107, 2015, Art. no. 142601.

[6] C. W. Bumby, R. A. Badcock, H. J. Sung, K. M. Kim, Z. Jiang, A. E. Pantoja, P. Bernado, M. Park, and R. G. Buckley, "Development of a brushless HTS exciter for a $10 \mathrm{~kW}$ HTS synchronous generator", Supercond. Sci. Technol. vol. 29, 2016, Art. ID 024008.

[7] Z. Jiang, C. W. Bumby, R. A. Badcock, H. J. Sung, N. J. Long, and N. Amemiya, "Impact of flux gap upon dynamic resistance of a rotating HTS flux pump," Supercond. Sci. Technol., vol. 28, Sep. 2015, Art. ID 115008.

[8] K. Ogasawara, K. Yasukochi, S. Nose, and H. Sekizawa, "Effective resistance of current-carrying superconducting wire in oscillating magnetic fields 1: Single core composite conductor," Cryoginics, vol. 16, pp. 3338, Jan. 1976.

[9] M. P. Oomen, J. Rieger, M. Leghissa, B. ten Haken, and H. H. J. ten Kate, "Dynamic resistance in a slab-like superconductor with $J_{\mathrm{c}}(B)$ dependence," Supercond. Sci. Technol., vol. 12, pp. 382-387, 1999.

[10] G. P. Mikitik and E. H. Brandt, "Generation of a de voltage by an ac magnetic field in type-II superconductors," Phys. Rew. B, vol. 64, Aug. 2001, Art. no. 092502.

[11] M. Ciszek, O. Tsukamoto, J. Ogawa, and D. Miyagi, "Energy losses in YBCO-123 coated conductors carrying transport current in perpendicular external magnetic field", AIP Conf. Proc. vol. 614, pp. 606-613, 2002.

[12] R. C. Duckworth, Y. F. Zhang, T. Ha, and M. J. Gouge "Dynamic resistance of YBCO-coated conductors in applied AC fields with DC transport currents and DC background fields," IEEE Trans. Appl. Supercond., vol. 21, pp. 3251-3256, June 2011.

[13] M. Iwakuma, K. Toyota, M. Nigo, T. Kiss, K. Funaki, Y. Iijima, T. Saitoh, Y. Yamada, and Y. Shiohara, "AC loss properties of YBCO superconducting tapes fabricated by IBAD-PLD technique," Physica C, vol. 412414, pp. 983-991, 2004.

[14] Z. Jiang, N. Amemiya, K. Kakimoto, Y. Iijima, T. Saitoh, and Y. Shiohara, "The dependence of AC loss characteristics on the space in stacked YBCO conductors," Supercond. Sci. Technol. vol. 21, 2008, Art. no. 015020.

[15] Z. Jiang, R. Toyomoto, N. Amemiya, X. Zhang, and C. W. Bumby "Dynamic resistance of a high-Tc coated conductor wire in a perpendicular magnetic field at 77 K," Supercond. Sci. Technol, vol. 30 , Jan. 2017, Art. no. 03LT01.

[16] Z. Jiang, R. Toyomoto, N. Amemiya, C. W. Bumby, R. A. Badcock, and N. J. Long, "Dynamic resistance measurements in a GdBCO coated condcutor," IEEE Trans. Appl. Supercond., vol. 27, no. 4, Jun. 2017, Art. no. 5900205 .

[17] Z. Jiang, W. Zhou, Q. Li, M. Yao, C. W. Bumby, M. Staines, R. A. Badcock, N. J. Long, J. Fang, and N. Amemiya, "Dynamic resistance characteristics in a YBCO wire carrying wide-range DC currents under AC magnetic fields with various directions," submitted to Supercond. Sci. Technol, 2017.

[18] W. Zhou, M. Staines, Z. Jiang, R. A. Badcock, N. J. Long, and J. Fang, "Shielding of Eddy Current Loss in Metal Layers of REBCO Superconducting tapes and Roebel Cables," Accepted for publication in IEEE Trans. Appl. Supercond., 2017.

[19] http://www.superpower-inc.com/system/files/SP_2G+Wire+Spec+Sheet_2014_web_v1_0.pdf

[20] S. Fukui, Y. Kitoh, T. Numata, O. Tsukamoto, J. Fujikami, and K. Hayashi, "Transport current AC losses of high-Tc superconducting tapes exposed to AC magnetic field," Advances in Cryogenic Engineering, vol. 44, pp. 723-730, 1998.

[21] Z. Jiang, K. P. Thakur, N. J. Long, R. A. Badcock, and M. Staines, "Comparison of transport AC losses in an eight-strand YBCO Roebel cable and a four-tape YBCO stack," Physica C, vol. 471, pp. 999-1002, 2011.

[22] E. H. Brandt and M. Indenbom, "Type-II-superconductor strip with current in a perpendicular magnetic field," Phys. Rew. B, vol. 48, pp. 12893 12906, Nov. 1993.

[23] V. Lahtinen, E. Pardo, J. Souc, M. Solovyov, and A. Stenvall, "Ripple field losses in direct current biased superconductors: Simulations and comparison with measurements," J. Appl. Phys. vol. 115, Mar. 2014, Art. no. 113907.

[24] V. Lahtinen and A. Stenvall, "The difficulty of modelling ripple field losses in superconductors using the eddy current model," IEEE Trans. Appl. Supercond., vol. 23, no. 3, Jun. 2013, Art. no. 4900505. 